\title{
Optimal Overcurrent Relay Coordination in Interconnected Networks by Using Fuzzy-Based GA Method
}

\author{
D. Solati Alkaran, M.R. Vatani, M.J. Sanjari, G.B. Gharehpetian, Senior, IEEE, and \\ M.S. Naderi
}

\begin{abstract}
A new objective function (OF) has been proposed for mathematical formulation of directional overcurrent (OC) relay coordination in interconnected networks. The fuzzy based Genetic Algorithm (GA) is applied to optimize the proposed OF for optimal coordination of $\mathrm{OC}$ relays. The defined fuzzy rules update the weighting factors of $O F$ during the simulation. The miscoordination problem of $\mathrm{OC}$ relays is solved while decreasing the operating time of the relays. The proposed method is implemented in three different networks and the simulation results have been compared with previous studies in order to illustrate the accuracy and efficiency of the proposed method to coordinate the directional $O C$ relays with both discrete and continuous time setting multipliers (TSMs). The results have also been compared with the results of other optimization methods. Considering the coefficients of $\mathrm{OC}$ characteristic curves as the $\mathrm{OF}$ variables ensures that the proposed method has no limitation for the types of characteristic curves which will be utilized. Presenting the new term in OF, the performance of the proposed method has not been affected by the size of the networks.
\end{abstract}

Index Terms--Overcurrent relay, optimal coordination, interconnected network, time interval coordination, genetic algorithm, Fuzzy logic.

\section{NOMENCLATURE}

OC Overcurrent relay

CTI Coordination time interval between main and backup relays

PSM Plug setting multiplier

TSM Time setting multiplier

OT Operation time

GA Genetic algorithm

OF Objective function

$\Delta t_{m b} \quad$ Coordination time between the main and backup relays

$t_{i}\left(x_{\text {close-in }}\right)$ Operation time of relay for the close-in fault

$I_{\text {Lmax }} \quad$ Maximum load current passing through the relay

$I_{p} \quad$ Minimum current detected as the fault current by the relay

$I_{F m i n} \quad$ Minimum fault current in the protection zone of the relay

D. Solati Alkaran, M. R. Vatani, M. J. Sanjari and G. B. Gharehpetian are with the Electrical Engineering Department, Amirkabir University of Technology, 15914 Tehran, Iran (e-mail: \{davoodsolati, mvatani, m_j_sanjari, grptian, salaynaderi\}@aut.ac.ir).

\begin{tabular}{|c|c|}
\hline$n$ & Number of relays \\
\hline$t_{i}$ & Operation time of relay for the close-in fault \\
\hline$t_{m}$ & $\begin{array}{l}\text { Operation time of main relay for close-in fault of } \\
\text { main relay }\end{array}$ \\
\hline$t_{b}$ & $\begin{array}{l}\text { Operation time of backup relay for close-in fault of } \\
\text { main relay }\end{array}$ \\
\hline $\mathrm{RCCC}$ & $\begin{array}{l}\text { Relay characteristic constant coefficients, } \\
\text { RCCC: }\{A, B, C, D\}\end{array}$ \\
\hline$a_{i}$ & Parameters of objective function $(i=1,2,3,4)$ \\
\hline$m b$ & $\begin{array}{l}\text { Number of pair relays including main and backup } \\
\text { relays }\end{array}$ \\
\hline $\bar{t}$ & Average of operation time of relays \\
\hline$\Delta \bar{t}_{-}$ & $\begin{array}{l}\text { Average of negative coordination time between the } \\
\text { main and backup relays }\end{array}$ \\
\hline$\Delta \bar{t}_{+}$ & $\begin{array}{l}\text { Average of positive coordination time between the } \\
\text { main and backup relays }\end{array}$ \\
\hline $\bar{n}_{-}$ & Average number of relays miscoordinations \\
\hline
\end{tabular}

\section{INTRODUCTION}

$\mathrm{T}$ HE main duty of protective relays is to detect the faults and clear them in minimum time by sending the "open" signal to the circuit breakers. OC relay is one of the most common because of its simplicity and economic features. It has been used as the main relay to protect the subtransmission and distribution systems or as backup protection of the transmission system [1]. Both the main and backup relays detect and sense the fault as soon as it occurs. However, the primary relay is the first one which should clear the fault and the backup relay would take over tripping with a predetermined time interval with the operation time (OT) of the main relay only if the primary relay does not eliminate the fault in its specific time [2]. The minimum OT of a backup relay should be more than the total of the related main relay OT, main circuit breaker OT, and overshoot time of the backup relay. Hence, the OC relays coordination can be considered a significant concern for power systems protection [3].

The settings of OC relays include plug setting multiplier (PSM) and time setting multiplier (TSM), which should be calculated such that the faulty part of the network is disconnected $[4,5]$. Generally, in traditional OC relays, the rating of PSM is from 50 to $200 \%$ in steps of $25 \%$ and the 
rating of TSM is from 0.05 to 1 in steps of 0.05 . However, these parameters can be set in a step of 0.01 in digital relays [6]. PSM is determined based on the minimum fault current and the maximum load current [7-9]. The coordination constraint between the main and backup relays can be

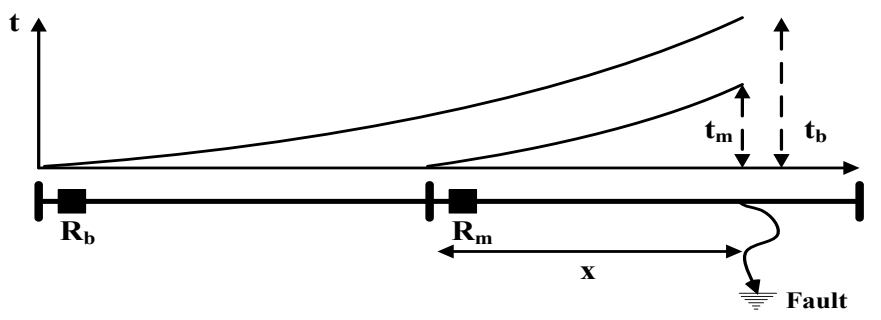

Fig. 1. Coordination constraint for a pair of main and backup relays

expressed as the following equation and is shown in Fig. 1 for a pair of main and backup relays:

$\Delta t_{m b}=t_{b}-t_{m}-C T I \geq 0$

The coordination of OC relays in a radial network is really simple, since each relay is the backup for its downstream relays, while in interconnected networks each relay may be a backup for more than one relay and also several relays can be the backup for one relay. Although OC relays have a simple structure, their coordination can be an intricate problem in some interconnected networks. This problem would become more complex if the size and complexity of the system increases. It should be noted that in the interconnected system the directional OC relays should be used, which is simply addressed by the OC relay in this paper. The traditional methods for overcurrent relays coordination in interconnected networks are time consuming considering the large amount of calculation burden and the difficulty of determining the power system break point. Diverse ranges of optimization methods and approaches have been proposed to achieve not only the coordination between the relays but also the optimal operation time of the protection system. The objective functions used in these methods have some drawbacks related to operation time of relays and their coordination.

The Genetic algorithm (GA) has a wide range of uses in power systems, one of which is the $\mathrm{OC}$ relays coordination in an interconnected network. In [10], GA based methods for optimal coordination of OC relays have been proposed. This method suffers from miscoordination $[6,11]$, meaning that the operation time of backup relays are less than the main ones. In this condition, the coordination between the main and backup relays is not satisfied and the backup relay operates sooner than the main relay.

The continuous GA method has been used for optimal coordination in $[12,13]$. However, this method has some drawbacks such as miscoordination. The other disadvantage related to this method is the way in which the value of TSM has been specified. Although the method claims that TSM is a continuous variable, it should be set in small steps even in modern and new digital relays. Therefore, by utilizing the continuous GA, the proposed methods in $[12,13]$ cannot find the optimum and best answer for discrete TSM without approximation or rounding the continuous answer to the nearest discrete one.

The presented method in [6] has solved the problem of determining the continuous and discrete TSM but the OT of the main and backup relays is large which can be harmful for electrical equipment because they have to tolerate the fault current for a longer time. This has a strong negative effect on the efficiency of the equipment and the rate of their aging.

The new objective function (OF) has been proposed in [14] to overcome the negative aspects of [6]. However, this problem has not been solved appropriately due to the formulation and coefficients of OF. In other words, the weighting factors of different parts of $\mathrm{OF}$ have been determined through the trial and error approach in [6] and [14]. An advanced coordination method for an optimized protection time grading is proposed in [15]. The mentioned study is carried out based on new nonstandard tripping characteristics for OC protection relays to achieve the highest possible reduction of tripping times for a selective fault clearing in distribution networks protected by OC relays without communication links. In [15], the OC relay coordination is translated to a mathematical optimization task, which is solved by the method of Lagrange generalized with the Karush-Kuhn-Tucker conditions. However, as stated in the subsection IV.A in this paper, considering TSMs as continuous variables in the process of $\mathrm{OC}$ relays coordination with discrete TSM leads to a near optimal solution for the mathematically formulated $\mathrm{OC}$ relay coordination.

A comprehensive study of meta-heuristic methods which are applied to coordinate overcurrent relays is presented in [16]. It expresses the procedures of utilizing the meta-heuristic methods in OC relays coordination. These methods include GA, particle swarm optimization (PSO), differential evolution (DE), harmony search (HS), and seeker optimization algorithm (SOA). Moreover, some comparisons among these methods have been made by simulating different scenarios. The results in [16] indicate that the DE and HS obtain a better answer than the other mentioned optimization methods. In addition, the PSO and SOA reach their best answers faster than the other methods. Although the paper has compared the different optimization methods, there is no explicit expression about the method of determining the OF weighting factors. Apart from [16], diverse types of optimization methods which are applied to coordinate overcurrent relays have been studied in [17] and [18]. In addition, the interior point method (IPM) as a mathematical optimization method has been used in [19] and the results have been compared with other OFs. The results have been compared with two categories of optimization methods including metaheuristic ones (GA and $\mathrm{DE}$ ) and hybrid ones (IPM-GA and IPM-DE). The proposed methods in [19] suffer from the similar drawback of [16]. In addition, the coefficients of characteristic curves have not been considered as the OF variables.

This paper presents a new accurate Fuzzy-GA based method for OC relays coordination in the interconnected network. The proposed method in this paper not only solves 
the problems of miscoordination and high OT of relays by defining a new OF, but also uses new constraints for better coordination between the relays. The newly defined OF leads to finding the best answer for both the continuous and discrete variables.

The main contributions of the proposed methods are listed as follows:

- A new expression of OF is proposed in this paper in order to overcome the drawbacks existing in previous methods, i.e. high value for the relays OT and using approximation in determining the discrete variables from the achieved continuous answer.

- The weighting factors for different terms of OF are calculated based on a Fuzzy logic procedure instead of the trial and error approach to determine the most accurate values for OF parameters.

- The newly proposed algorithm is able to minimize the number of miscoordinations in large networks in which all constraints cannot be satisfied by using previous methods.

- The characteristic curves coefficients of OC relays and PSMs are considered as the optimization problem variables to enhance the efficiency and flexibility of protection coordination in the proposed method. As a result, the best curve can be achieved for each main and backup relay. Therefore, there is no limitation for choosing or defining diverse kinds of characteristic curves in modern OC relays.

- The objective function presented in this paper uses the average operation time of the OC relays as well as average $\Delta t$ between the main and backup relays. This noticeably decreases the dependency of the proposed method performance upon the size of the network utilized for simulation.

The rest of the paper is organized as follows: the problem formulation is described in Section III. Section IV explains the proposed method and its main concepts. Simulation results and their discussion are presented in section V. Finally, the conclusion is drawn in Section VI.

\section{PROBLEM FoRMULATION}

The OC relays coordination in interconnected networks is complex due to the fact that there are a large number of constraints stated in the form of (1). Considering the fact that there is a low chance to coordinate the $\mathrm{OC}$ relays in an optimal way using the traditional methods, the coordination problem of OC relays in an interconnected network changes into an optimization problem with a large number of constraints.

There are three main points in the setting and coordination of OC relays, high speed operation of relay, selectivity which means that each relay should operate only for the faults occurring in the corresponding protection zone of the relay and reliability which means each relay must be appropriately backed up by some other relays. The mentioned concerns are considered in this paper to enhance the dependability of the protection system and consequently the security of the power system $[20,21]$.

In an interconnected network with a lot of OC relays, if the
OF is to optimize the operation speed of each relay separately, the problem will be very complex and practically insoluble. Hence, the overall speed operation of all relays should be considered. The following equation can be introduced to satisfy the OF for this consideration:

$$
O F=\sum_{i=1}^{n} t_{i}\left(x_{\text {close-in }}\right)
$$

The OC relays should be directional, i.e. each of them operates only for its protection zone. This means that they should detect faults only occurring in front of the relay. Moreover, the PSM of these relays should be properly set. This setting is defined as the minimum current which can be detected as the fault current by the relay. This current should be more than the maximum load current passing through the relay multiplied by a certainty factor (usually 1.3) and less than the minimum current for the fault occurring in the protection zone of the relay. This relation is expressed as follows:

$$
1.3 I_{L \max } \leq I_{p} \leq I_{F \min }
$$

In addition, the minimum coordination time interval should be satisfied between the main and backup relays to ensure their selective operation. Considering all the above explanations, it can be concluded that for setting and coordination of OC relays in an interconnected network, the following optimization problem should be solved:

$$
\begin{array}{ll}
\text { Minimize } & O F=\sum_{i=1}^{n} t_{i} \\
\text { subject to: } & \Delta t_{j}=t_{b_{j}}-t_{m_{j}}-C T I>0 \\
& 1 \leq j \leq m b \\
& I_{p \min } \leq I_{p} \leq I_{p \max } \\
& T_{\text {m }} \leq T S M \leq T S M_{\max }
\end{array}
$$

The OT of a relay is related to its RCCC in terms of its setting parameters and the fault current flowing through the relay. Generally, the equation of the relay curve can be expressed as follows:

$$
t=\left(\frac{A}{\left(I / I_{p}\right)^{B}-C}+D\right) T S M
$$

In order to solve (4), both the mathematical methods and the intelligent searching algorithm methods can be used. Considering the large amount of inequality constraints in this optimization problem, the mathematical methods are not effective, while the intelligent searching methods can be a practical solution. In modern digital OC relays, PSM, TSM, and the relay characteristic curves coefficients can be set in very small steps. Consequently, the variables of the optimization problem can be discrete and continuous. The Genetic algorithm (GA) is one of the most robust tools for solving such a mixed integer optimization problem. Most intelligent searching algorithms solve the discrete problems in a continuous way and the answers are rounded to the nearest discrete variable at the end of each generation, while GA can 
discretely solve the optimization problem with discrete variables during the solving process. This is the reason why the GA is used in this paper to solve the optimization problem. In order to use the GA, at first an OF should be defined appropriately including the related constraints. These constraints should be added as a penalty value to the OF in order to decrease the probability of selecting the chromosomes which do not satisfy the constraints by increasing the OF for them. Consequently, the final OF can be implemented as follows:

$$
O F=\sum_{i=1}^{n} a_{i} f\left(t_{i}\right)+\sum_{j=1}^{m b} b_{j} g\left(\Delta t_{j}\right)
$$

where, $a_{i}$ and $b_{j}$ are the constant coefficient as weighting factors.

Finally, GA chooses some chromosomes for crossover, mutation, and selection operators in proportion to their fitness. These operators generate a new population and it will be repeated until reaching the stop condition.

\section{Proposed Method}

\section{A. Continuity or Discreteness of TSM}

In many studies, TSMs are considered as continuous variables in the process of the $\mathrm{OC}$ relays coordination with discrete TSM. Then, the feasible answers are achieved by rounding the continuous results to the nearest discrete TSM at the end of each generation or at the end of the program. This process has two main drawbacks:

- The number of possible states and the search space would be increased if the TSMs are considered as the continuous variables, while the answer can be found in a smaller discrete space. Therefore, the number of iterations for convergence is decreased. Moreover, in large networks, the search space may be so wide that it results in divergence of the optimization program.

- The relation between the fitness function and the problem variables is not clear or obvious in the scenario in which the optimization method works with continuous TSM and round it after optimization. Thus, it is a possible condition in which a global optimum is achieved by continuous TSMs but it would not be the global optimum among the discrete scenarios after rounding to a discrete TSM. As shown in Fig. 2, $K_{0}$ is a global minimum value considering continuous TSMs while $K_{1}, K_{2}$, and $K_{3}$ are the possible solution considering discrete TSMs. As shown in Fig. 2, it is obvious that if the continuous values of TSMs are considered, the optimization method is converged to the answer $K_{0}$ as the minimal value of the $\mathrm{OF}$ in continuous space. After rounding $K_{0}$ to a discrete variable, either $K_{l}$ or $K_{2}$ is obtained in the discrete space. However, scenario $K_{3}$ is the answer among the discrete scenarios and has a considerable difference with $K_{1}$ and $K_{2}$.

According to the aforementioned issues, in this paper, a discrete gene is considered as a binary string in GA for the relay with the discrete TSM and a continuous gene as a real number for the continuous variables. Therefore, the searching space becomes smaller and the chance for obtaining the correct answer is enhanced because the achieved optimal TSM is not rounded to the nearest discrete TSM.

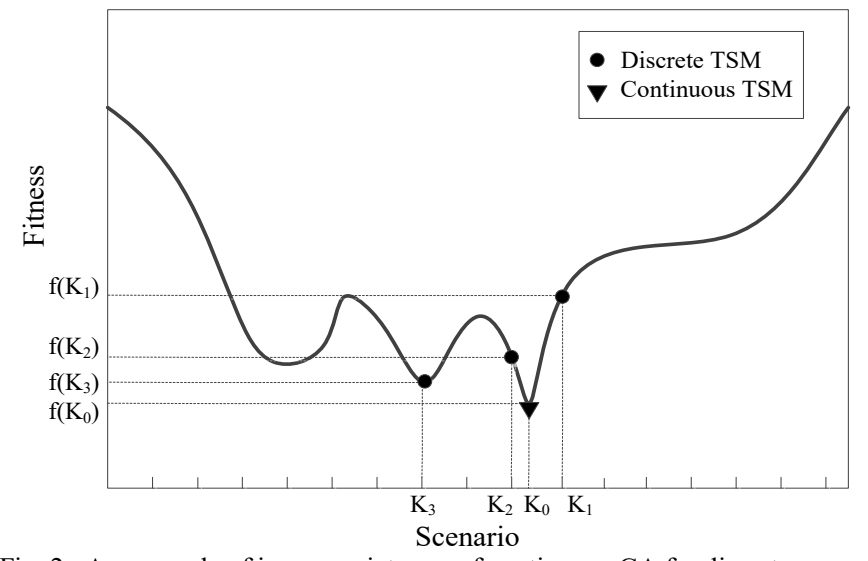

Fig. 2. An example of inappropriate use of continuous GA for discrete variables

\section{B. Fitness Function}

The following OF was proposed in [6] to coordinate the $\mathrm{OC}$ relays:

$$
O F=\alpha_{1} \sum_{i=1}^{n}\left(t_{i}\right)^{2}+\alpha_{2} \sum_{j=1}^{m b}\left(\Delta t_{j}-\beta\left(\Delta t_{j}-\left|\Delta t_{j}\right|\right)\right)^{2}
$$

By comparing (7) and (6), it can be inferred that the main function in [6] has been defined as $f\left(t_{i}\right)=\left(t_{i}\right)^{2}$. In addition, the constraint has been added to the OF as $g\left(\Delta t_{j}\right)=\left(\Delta t_{j}-\beta\left(\Delta t_{j}-\left|\Delta t_{j}\right|\right)\right)^{2}$. The presented OF in [6] has the following disadvantages:

- The main objective of the setting and coordination of OC relays in interconnected networks is to minimize the total OT of the relays. In this regard, using the square of OT in the OF leads to a distraction from the optimal answer. For more clarification, two scenarios are given as an example. It is assumed that the OT of the $i$-th and $j$-th relays are 0.1 and 0.9 for the first scenario and the similar variables for the second scenario are 0.4 and 0.813 , respectively. The value of the main function is 0.82 for both scenarios (using $\left.f\left(t_{i}\right)=\sum_{i=1}^{n}\left(t_{i}\right)^{2}\right)$,

while it is obvious that the first scenario presents the better answer because the average OT of the OC relay in the first scenario is less than the second one $(0.5$ and 0.6065 , respectively). To correct the mentioned problem of the main function, this paper uses the $f\left(t_{i}\right)=t_{i}$ as the main OF.

- The penalty function presented in [6] is as $g\left(\Delta t_{j}\right)=\Delta t_{j}^{2}$, which has the same drawback above because of using the square of $\Delta t_{j}$, especially when this square value is applied in large networks which have more constraints. This problem usually creates the local optimums in the OF. In this condition, the probability of pining GA in these local optimums is enhanced. Thus, a linear penalty function as $g\left(\Delta t_{j}\right)=\left|\Delta t_{j}\right|$ is proposed in this paper. Furthermore, the penalty function for both positive and negative $\Delta t$ are defined separately with 
different coefficients as (8) since eliminating negative $\Delta t \mathrm{~s}$ is more important than minimizing the positive ones.

$$
\begin{aligned}
& g\left(\Delta t_{j}\right)=b_{1} g_{1}\left(\Delta t_{j}\right)+b_{2} g_{2}\left(\Delta t_{j}\right) \\
& g_{1}\left(\Delta t_{j}\right)=\left|\Delta t_{j}\right|-\Delta t_{j} \\
& g_{2}\left(\Delta t_{j}\right)=\left|\Delta t_{j}\right|+\Delta t_{j}
\end{aligned}
$$

- In large networks with numerous OC relays, the number of constraints for the coordination problem is really high and in some conditions, there is no feasible answer in which all the main and backup relays are coordinated. In other words, it is possible that some constraints are not satisfied in the optimal solution. The number of constraints is important because additional costs should be paid for alternate backup protection for each of the relays related to the unsatisfied constraints. Thus, in order to reduce the number of unsatisfied constraints, the following function is proposed in this paper to add to the penalty function:

$$
h\left(\Delta t_{j}\right)=\operatorname{sgn}\left(\left|\Delta t_{j}\right|-\Delta t_{j}\right)= \begin{cases}1 & \Delta t_{j}<0 \\ 0 & \Delta t_{j} \geq 0\end{cases}
$$

where the $\operatorname{sgn}(x)$ is the sign function which is equal to $-1,1$ or 0 if $x$ is negative, positive or zero, respectively.

As an example, suppose that there is a network with three OC relays which are coordinated by using two different scenarios. Table I shows the supposed value of $\Delta t$ s obtained from these scenarios. As shown in Table I, there are 3 and 1 unsatisfied constraints by using the first and the second scenario, respectively.

Considering the penalty function as $g(\Delta t)=\Delta t^{2}$, the penalty values for these scenarios are 0.0075 and 0.6536 , respectively. Based on the proposed method in [6], the results of the first scenario are considered as the answer, while it is obvious that the results of the second scenario are better because of its less unsatisfied constraints. However, considering the proposed penalty function in this paper, the penalty value for these two scenarios are 3.15 and 1.96, respectively. Thus, the proposed penalty function in this paper is more accurate than the previous methods such as [6].

Considering the above mentioned issues, the proposed OF is defined as follows in this paper to be minimized by GA:

$$
\begin{gathered}
O F=a_{1} \frac{\sum_{i=1}^{n} t_{i}}{n}+a_{2} \frac{\sum_{j=1}^{m b}\left(\left|\Delta t_{j}\right|-\Delta t_{j}\right)}{\max \left(1, \sum_{j=1}^{m b} \operatorname{sgn}\left(\left|\Delta t_{j}\right|-\Delta t_{j}\right)\right)}+ \\
a_{3} \frac{\sum_{j=1}^{m b}\left(\left|\Delta t_{j}\right|+\Delta t_{j}\right)}{m b-\sum_{j=1}^{m b} \operatorname{sgn}\left(\left|\Delta t_{j}\right|-\Delta t_{j}\right)}+a_{4} \frac{\sum_{j=1}^{m b} \operatorname{sgn}\left(\left|\Delta t_{j}\right|-\Delta t_{j}\right)}{m b}
\end{gathered}
$$

Each term in the proposed OF is normalized with respect to the number of non-zero terms in each summation to decrease the dependency of coefficients on the number of relays and coordination constraints as well as to determine the thresholds of coefficients in the Fuzzy process.

TABLE I

$\triangle T$ FOR TWO SUPPOSED SCENARIOS

\begin{tabular}{|c|c|c|}
\hline$\Delta t$ & $\begin{array}{c}\text { Results of the First } \\
\text { Assumed Method }\end{array}$ & $\begin{array}{c}\text { Results of the Second } \\
\text { Assumed Method }\end{array}$ \\
\hline 1 & -0.05 & 0.06 \\
\hline 2 & -0.05 & 0.10 \\
\hline 3 & -0.05 & -0.80 \\
\hline
\end{tabular}

\section{Determination of Fitness Function Coefficients}

The OF stated in (7) has three coefficients, $\alpha_{1}, \alpha_{2}$ and $\beta$ which are constant and defined based on the trial and error approach in [6]. However, they are highly related to the nature of the problem and under-studied network. Therefore, they should be determined separately in each network. Moreover, since these coefficients are constant during the optimization procedure, GA may converge to a local optimal point and the global optimum is missed.

The qualifiers such as very small, small, average, large, and very large (i.e., SS, S, N, L, and LL, respectively) are used to describe the Fuzzy rules which are the relationships between the inputs and outputs of a Fuzzy function. In this regard, this paper proposes a Fuzzy logic with the rules listed in Table II. Some of these rules are derived based on the mathematical expression of (10).

The proposed OF in (10) has four parameters, $a_{l}$ influences the value of relays OT which is shown as $\bar{t}$ in Table II, $a_{2}$ has an effect on negative $\Delta t s$ which is shown as $\Delta \bar{t}_{-}$in Table II, $a_{3}$ has an impact on large value of $\Delta t s$ which is shown as $\Delta \bar{t}_{+}$in Table II, and $a_{4}$ decreases the number of unsatisfied constraints which is shown as $\bar{n}_{-}$in Table II. However, the other Fuzzy rules have been derived based on observation of input-output behaviors. As an example and further clarification about the interpretation of Fuzzy functioning in this paper, the first row of this table expresses that if $\bar{t}$ (the average of relays operation time) is a large value and $\bar{n}_{-}$(the number of constraints which are not satisfied) is a very small value, the following measures should be taken without considering the values of $\Delta \bar{t}_{-}$and $\Delta \bar{t}_{+}$:

- The value of $a_{l}$ should be very large

- The value of $a_{3}$ should be very small

- The value of $a_{2}$ and $a_{4}$ should remain without any change.

As a cycle, the proposed Fuzzy logic determines the parameters $a_{1}, a_{2}, a_{3}$, and $a_{4}$ considering the outputs of GA after a preset number of GA iterations which is called maximum Fuzzy generation (e.g., 50 iterations). In other words, for example, GA is run for 50 iterations. Then, Fuzzy logic uses the outputs of GA after these iterations to reproduce the OF parameters through the Fuzzy rules. This procedure will be repeated after each of the 50 -iterations to reach the maximum number of generations. 
TABLE II

THE RULES OF FUZZY LOGIC

\begin{tabular}{|c|c|c|c|c|c|c|c|}
\hline $\bar{t}$ & $\Delta \bar{t}_{-}$ & $\Delta \bar{t}_{+}$ & $\bar{n}_{-}$ & $a_{1}$ & $a_{2}$ & $a_{3}$ & $a_{4}$ \\
\hline $\mathrm{L}$ & $* *$ & $* *$ & $\mathrm{SS}$ & LL & $* *$ & $\mathrm{SS}$ & $* *$ \\
\hline $\mathrm{L}$ & $* *$ & $* *$ & $\mathrm{~S}$ & $\mathrm{~L}$ & $* *$ & $\mathrm{SS}$ & $* *$ \\
\hline $\mathrm{L}$ & $* *$ & $* *$ & $\mathrm{~L}$ & $\mathrm{~S}$ & $* *$ & SS & $* *$ \\
\hline $\mathrm{N}$ & $* *$ & $* *$ & $\mathrm{SS}$ & $\mathrm{L}$ & $* *$ & $\mathrm{~S}$ & $* *$ \\
\hline $\mathrm{N}$ & $* *$ & $* *$ & $\mathrm{~S}$ & $\mathrm{~N}$ & $* *$ & $\mathrm{~S}$ & $* *$ \\
\hline $\mathrm{N}$ & $* *$ & $* *$ & $\mathrm{~L}$ & SS & $* *$ & $\mathrm{~S}$ & $* *$ \\
\hline $\mathrm{S}$ & $* *$ & $* *$ & $* *$ & $\mathrm{~N}$ & $* *$ & $* *$ & $* *$ \\
\hline $\mathrm{SS}$ & $* *$ & ** & $* *$ & $\mathrm{~S}$ & $* *$ & $* *$ & $* *$ \\
\hline SS & $* *$ & $\mathrm{~L}$ & $* *$ & $* *$ & $* *$ & LL & $* *$ \\
\hline $\mathrm{S}$ & $* *$ & $\mathrm{~L}$ & $* *$ & $* *$ & $* *$ & $\mathrm{~L}$ & $* *$ \\
\hline $\mathrm{N}$ & $* *$ & $\mathrm{~L}$ & $* *$ & $* *$ & $* *$ & $\mathrm{~N}$ & $* *$ \\
\hline $\mathrm{L}$ & $* *$ & $\mathrm{~L}$ & $* *$ & $* *$ & $* *$ & $\mathrm{SS}$ & $* *$ \\
\hline $\mathrm{SS}$ & $* *$ & $\mathrm{~N}$ & $* *$ & $* *$ & $* *$ & LL & $* *$ \\
\hline $\mathrm{S}$ & $* *$ & $\mathrm{~N}$ & $* *$ & $* *$ & $* *$ & $\mathrm{~N}$ & $* *$ \\
\hline $\mathrm{N}$ & $* *$ & $\mathrm{~N}$ & $* *$ & $* *$ & $* *$ & $\mathrm{~N}$ & $* *$ \\
\hline $\mathrm{L}$ & $* *$ & $\mathrm{~N}$ & $* *$ & $* *$ & $* *$ & SS & $* *$ \\
\hline$* *$ & $* *$ & $\mathrm{~S}$ & $* *$ & $* *$ & $* *$ & $\mathrm{~S}$ & $* *$ \\
\hline$* *$ & $* *$ & $\mathrm{SS}$ & $* *$ & $* *$ & $* *$ & $\mathrm{~S}$ & $* *$ \\
\hline$* *$ & $\mathrm{~L}$ & $* *$ & $* *$ & $* *$ & $\mathrm{LL}$ & $* *$ & $* *$ \\
\hline$* *$ & $\mathrm{~N}$ & $* *$ & $* *$ & $* *$ & $\mathrm{~N}$ & $* *$ & $* *$ \\
\hline$* *$ & $\mathrm{~S}$ & $* *$ & $* *$ & $* *$ & $\mathrm{~S}$ & $* *$ & $* *$ \\
\hline$* *$ & SS & $* *$ & $* *$ & $* *$ & $\mathrm{~S}$ & $* *$ & $* *$ \\
\hline$* *$ & $* *$ & $* *$ & $\mathrm{SS}$ & $* *$ & $* *$ & $* *$ & SS \\
\hline$* *$ & $* *$ & $* *$ & $\mathrm{~S}$ & $* *$ & $* *$ & $* *$ & $\mathrm{~L}$ \\
\hline$* *$ & $* *$ & $* *$ & $\mathrm{~L}$ & $* *$ & $* *$ & $* *$ & $\mathrm{LL}$ \\
\hline
\end{tabular}

\section{Coefficients of Characteristic Curves}

Most papers presented in the field of OC relays coordination have considered only the TSM of relays as the optimization variable $[6,11]$. However, some papers have considered the types of standard characteristic curves as the variables of their OFs [22]. In modern digital relays, some variables can be set almost as continuous ones in a defined range. In this regard, [22] has not taken benefits of this important feature of the modern digital relays because it has considered all of their variables as discrete ones. The equation of the characteristic curves can be expressed as (5). Therefore, the types of curves are predetermined based on their specific coefficients, while these coefficients can be set in modern digital relays [23]. These coefficients are considered as variables in the proposed OF. This point ensures that the proposed method does not have any limitation for using the different kinds of characteristic curves in its algorithm.

The objective function presented in this paper uses the average operation time of the OC relays as well as average $\Delta t$ between the main and backup relays. However, the objective functions in the other articles use the sum of operation time of OC relays and sum of $\Delta t \mathrm{~s}$. This is the reason why the coefficients of the objective functions in those methods are strongly dependent upon the number of the relays utilized in a network. Moreover, these methods use the trial and error approach for determining the coefficients of their objective functions. Therefore, when they are applied to a larger network, the chance of finding the optimum answer decreases; hence the calculation time increases. On the other hand, the coefficients of the objective function proposed in this paper are modified during the simulation process.

The following procedure explains the steps of the proposed method when it is applied to a network regardless of its size.

Step 1: Data acquisition of the network such as transmission lines, generators, and transformers.

Step 2: Load flow study for determining the maximum load currents for each relay.

Step 3: Determining the pairs of primary and backup relays.

Step 4: Calculation of the minimum fault current $\left(I_{F m i n}\right)$ and maximum fault current $\left(I_{F \max }\right)$ flowing through each relay based on the proposed method in [24] using impedance matrix $Z_{\text {bus }}$ of the network.

Step 5: Determining the allowable range for the PSM and TSM of each OC relay based on the catalog instruction and according to (4).

Step 6: Determining the characteristic curves of each OC relay. Then, each type of these curves is numbered and the function and the operation time will be defined. For example, assume that there are four types of characteristic curves. Table III indicates the tag of each curve for this example.

TABLE III

EXAMPLE OF NUMBERING THE CHARACTERISTIC CURVES

\begin{tabular}{|c|c|}
\hline Type of Curve & Tag Number \\
\hline IEC Normally Inverse & 1 \\
\hline IEC Very Inverse & 2 \\
\hline IEEE Extremely Inverse & 3 \\
\hline Inverse User-Defined & 4 \\
\hline
\end{tabular}

Then, the function of operation time for each OC relay will be defined. With respect to Table III, the operation time of OC relay in IEC Normally Inverse curve, which is called $f_{l}$, can be written as follows:

$$
t=\frac{0.14 \times T S M}{(P S M)^{0.02}-1}
$$

Step 7: Calculation of the maximum faults currents flowing through each pair of primary and backup relays as a close-in fault of the primary relay.

Step 8: Determining the problem variables for creating the chromosomes in GA as shown in Fig. 3.

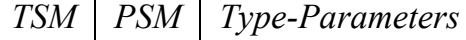

Fig. 3. The defined chromosome in the proposed method

where, $T S M=\left[T S M_{l}, T S M_{2}, \ldots, T S M_{n}\right]$ and $P S M=\left[P S M_{l}\right.$, $P S M_{2}$, . . . ., $\left.P S M_{n}\right]$ and Type-Parameters $=[$ Type-

Parameters $_{1}$, Type-Parameters ${ }_{2}$, . . ., Type-Parameter $\left.s_{n}\right]$. Type-Parameters ${ }_{n}$ includes the defined number of each characteristic curve based on step 6 and its coefficients. For instance, assume an inverse user-defined characteristic curve, which has been numbered 4 in step 6 . This curve has an equation similar to (5). Then, the variable Type-Parameters for the assumed curve is given below:

$$
\text { Type-Parameters }=[4, A, B, C, D]
$$


Step 9: This is the final step. In this step, the GA starts to work with the defined chromosomes in step 8. After a predefined number of iterations, the weighting factors will be updated through the defined fuzzy rules and the GA starts to work with the new weighting factors. It will be repeated until reaching the best answer.

It is obvious that steps 1-8 are not affected by the size of the network. Sometimes, these data could be prepared before starting the main algorithm.

The final step (i.e., step 9) uses fuzzy rules and GA both of which are general tools for applying to different kinds of problems. However, applying GA to a larger network with more variables will increase the simulation time but the accuracy of the proposed method will not decrease.

The proposed method procedure is shown in the flowchart of Fig. 4. In this figure, $G$ is the number of iterations and $F$ is the preset number of GA iterations before each usage of Fuzzy logic.

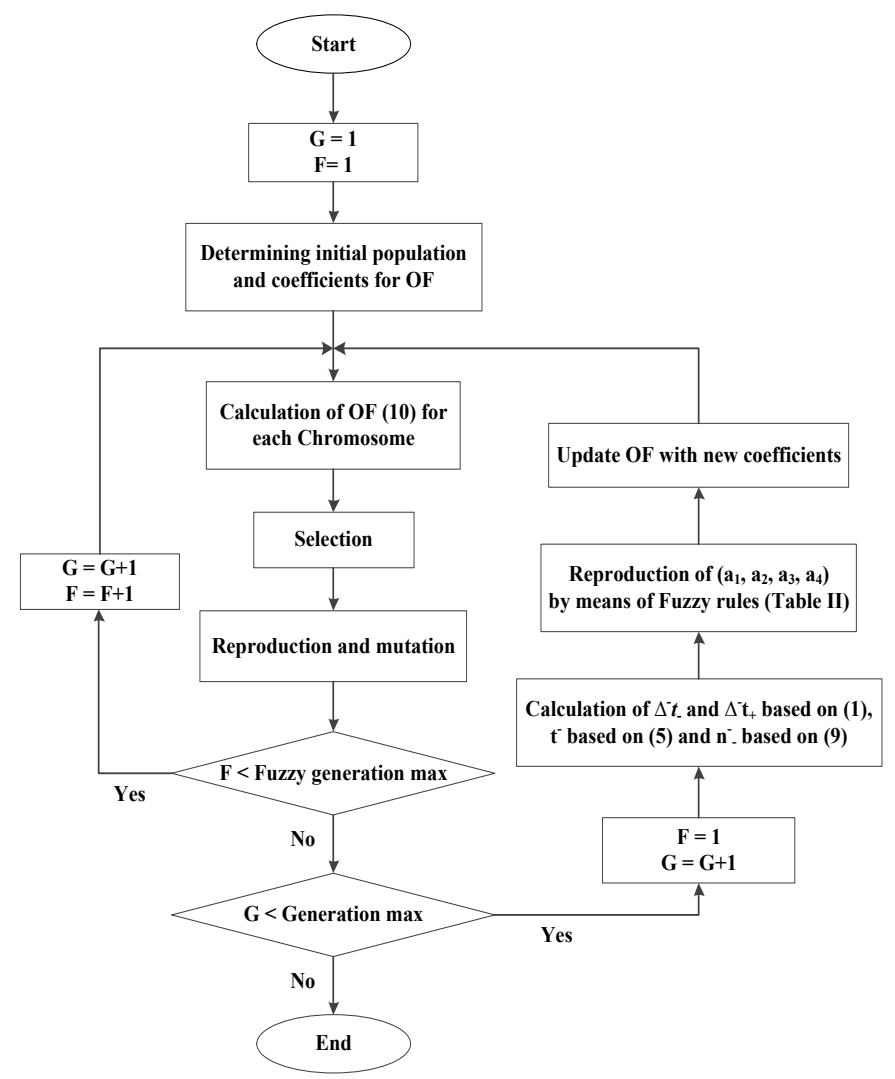

Fig. 4. Flowchart of the proposed method

\section{SimUlation Results AND Discussion}

In order to investigate the accuracy and efficiency of the proposed method, it is applied to three different networks including 6-bus, 8-bus and IEEE 30-bus test systems. In addition, three scenarios are defined. The results of the proposed method are compared with [6] in the first and second scenarios and with [19] in the third scenario. In the first scenario, PSMs and relays operation characteristics are defined similar to [6] because the best comparison can be achieved when the parameters are the same for both methods. Moreover, by considering the similar parameters with [6], the efficiency of the proposed OF in [6] and this paper can be compared precisely. The additional optimization variables such as PSM and the coefficients of characteristic curves are considered in the second scenario to show the best operation of the proposed method. In the third scenario, the proposed method in this paper is compared with the presented methods in [19]. These methods include different types of optimization methods which have been applied to the problem of coordination of OC relays in interconnected networks.

The values of maximum load current have been obtained through MATLAB software. The test systems have been simulated in the mentioned software based on the given data in [24] and [25] for the first and second test systems, respectively. Then, the load flow program is run and the values of maximum load current are achieved.

The values of minimum and maximum fault currents have been determined by the proposed method in [24]. The paper presents a new accurate method for determining the critical fault point based on which the maximum and minimum values of fault current can be obtained. This method analytically calculates the impedance matrix $Z_{\text {bus }}$ of the network. The critical fault point is determined through the calculated impedance matrix. In the proposed method, a hypothetical bus $X$ is considered as the fault location in each line of an interconnected network. Then, it is moved from the beginning to the end of each line and impedance matrix $Z_{b u s}$ will be calculated for each of these movements. In order to update the $Z_{\text {bus }}$ for each step, the admittance matrix $Y_{\text {bus }}$ of the network is applied. The $Y_{b u s}$ is used because of its simple rules for construction. Finally, the critical fault point is determined for each line and the values of maximum and minimum fault currents will be obtained.

\section{A. Scenario 1}

In this scenario, the proposed method is applied on an 8-bus network. In this scenario, the optimization variable is just TSM to show the efficiency of the proposed expression of OF and its weighting factors in comparison with the presented methods in [6]. The network and its data can be found in [24]. The weighting factors of OF obtained by Fuzzy rules are listed in Table IV. TSM, PSM, and OT of the relays in this network are listed in Table $\mathrm{V}$ for both proposed methods in this paper and [6]. As shown in Table V, the proposed method in this paper results in decreasing the TSM and the OT of the relays compared to [6].

The current of close-in fault for each pair of relays are listed in Table VI by using the proposed method in this paper. Also, the value of $\Delta t$ achieved by the proposed method in this paper and [6] is shown in this table. These results illustrate that the proposed method in this paper not only decreases the OT of the relays but also keeps the coordination of all their pairs.

Better results are achieved since the coefficients are determined through Fuzzy logic. Fig. 5 shows these coefficients variations during the Fuzzy process. It is obvious 
that the mentioned coefficients are converged to their optimum value considering the defined rules.
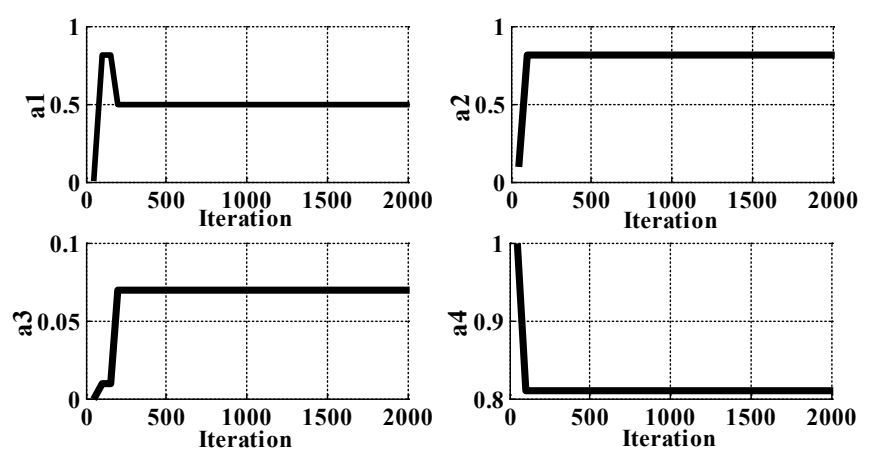

Fig. 5. Convergence of OF's coefficients in the first scenario

TABLE IV

THE PROPOSED OF'S COEFFICIENTS IN THE FIRST SCENARIO

\begin{tabular}{|c|c|}
\hline Weighting Factor & Value \\
\hline$a_{1}$ & 0.50 \\
\hline$a_{2}$ & 0.81 \\
\hline$a_{3}$ & 0.70 \\
\hline$a_{4}$ & 0.81 \\
\hline
\end{tabular}

TABLE V

PSM, TSM, AND OT OF THE RELAYS IN THE FIRST SCENARIO

\begin{tabular}{|c|c|c|c|c|c|c|}
\multicolumn{1}{c|}{$\begin{array}{c}\text { Relay } \\
\text { No. }\end{array}$} & $\begin{array}{c}\text { PSM } \\
\text { (A) }\end{array}$ & $\begin{array}{c}\text { TSM } \\
(\mathrm{s})\end{array}$ & $\begin{array}{c}\text { OT } \\
\text { (s) }\end{array}$ & $\begin{array}{c}\text { PSM } \\
\text { (A) }\end{array}$ & $\begin{array}{c}\text { TSM } \\
(\mathrm{s})\end{array}$ & $\begin{array}{c}\text { OT } \\
(\mathrm{s})\end{array}$ \\
\hline 1 & 500 & 0.15 & 0.5894 & 500 & 0.1 & 0.3929 \\
\hline 2 & 800 & 0.35 & 1.2173 & 800 & 0.3 & 1.0434 \\
\hline 3 & 600 & 0.25 & 0.9621 & 600 & 0.25 & 0.9621 \\
\hline 4 & 800 & 0.1 & 0.6636 & 800 & 0.1 & 0.6636 \\
\hline 5 & 550 & 0.1 & 0.766 & 550 & 0.05 & 0.383 \\
\hline 6 & 550 & 0.25 & 0.7623 & 550 & 0.2 & 0.6099 \\
\hline 7 & 650 & 0.2 & 0.7059 & 650 & 0.2 & 0.7059 \\
\hline 8 & 550 & 0.25 & 0.7625 & 550 & 0.2 & 0.61 \\
\hline 9 & 540 & 0.05 & 0.3479 & 540 & 0.05 & 0.3479 \\
\hline 10 & 550 & 0.15 & 0.6883 & 550 & 0.15 & 0.6883 \\
\hline 11 & 650 & 0.25 & 0.9834 & 650 & 0.25 & 0.9834 \\
\hline 12 & 550 & 0.4 & 1.1847 & 550 & 0.4 & 1.1847 \\
\hline 13 & 600 & 0.1 & 0.4665 & 600 & 0.1 & 0.4665 \\
\hline 14 & 800 & 0.15 & 0.5944 & 800 & 0.15 & 0.5944 \\
\hline Sum & & 2.75 & 10.694 & & 2.5 & 9.636 \\
\hline
\end{tabular}

B.

\section{Scenario 2}

In this scenario, the IEEE 30-bus test system is simulated to show the proposed method effectiveness in optimal relay coordination. The network and its data can be found in [25]. In this scenario, TSM, PSM, and the coefficients of characteristic curves are considered as the optimization variables. The proposed OFs stated in this paper and [6] are simulated in this scenario. The weighting factors of OF obtained from the fuzzy controller are listed in Table VII. TSM, PSM, and OT of the relays in this network are listed in Table VIII for both OFs proposed in this paper and [6]. Moreover, the coefficients of characteristic curves obtained from both OFs are listed in this table. However, the value of $D$ is considered to be equal to zero similar to IEC standards [26]. The value of $\Delta t$ for the proposed methods in this paper and [6] is shown in Table IX. This table indicates that there are 17 miscoordinations in the results obtained from [6], while the number of miscoordinations is only two by using the proposed OF in this paper because of adding a new term to the $\mathrm{OF}$ as explained in section IV.B which diminishes the number of miscoordinations.

TABLE VI

Close-in Fault Current Value for Each Pair of Relays and $\Delta \mathrm{T}$ FOR EACH PAIR OF RELAYS IN THE FIRST SCENARIO

\begin{tabular}{|c|c|c|c|c|c|}
\hline $\begin{array}{c}\text { Main } \\
\text { relay }\end{array}$ & $\begin{array}{c}\text { Backup } \\
\text { relay }\end{array}$ & $\begin{array}{c}\text { Main relay } \\
\text { close-in } \\
\text { fault } \\
\text { current } \\
\text { (kA) }\end{array}$ & $\begin{array}{c}\text { Backup } \\
\text { relay } \\
\text { close-in } \\
\text { fault } \\
\text { current } \\
\text { (kA) }\end{array}$ & $\begin{array}{c}\Delta \mathrm{t} \\
\text { Proposed } \\
\text { in [6] (s) }\end{array}$ & $\begin{array}{c}\Delta \mathrm{t} \\
\text { Proposed } \\
\text { method } \\
\text { (s) }\end{array}$ \\
\hline 8 & 7 & 4.9618 & 1.5209 & 0.4673 & 0.6198 \\
\hline 2 & 7 & 5.3623 & 1.5281 & 0.0028 & 0.1767 \\
\hline 2 & 1 & 5.3623 & 0.8049 & 0.6290 & 0.0541 \\
\hline 3 & 2 & 3.3345 & 3.3345 & 0.2675 & 0.0332 \\
\hline 4 & 3 & 2.2343 & 2.2343 & 0.2057 & 0.2057 \\
\hline 5 & 4 & 1.3529 & 1.3529 & 0.1885 & 0.5715 \\
\hline 6 & 14 & 4.965 & 1.5229 & 0.4825 & 0.635 \\
\hline 14 & 1 & 4.2327 & 0.7941 & 1.3181 & 0.5473 \\
\hline 1 & 6 & 2.6825 & 2.6825 & 0.0532 & 0.0411 \\
\hline 9 & 10 & 1.4437 & 1.4437 & 0.3173 & 0.3173 \\
\hline 10 & 11 & 2.3347 & 2.3347 & 0.2195 & 0.2195 \\
\hline 11 & 12 & 3.4808 & 3.4808 & 0.0492 & 0.0492 \\
\hline 12 & 14 & 5.3651 & 1.5294 & 0.0489 & 0.0489 \\
\hline 12 & 13 & 5.3651 & 0.8056 & 0.8135 & 0.8135 \\
\hline 13 & 8 & 2.4907 & 2.4907 & 0.2292 & 0.0101 \\
\hline 7 & 13 & 4.2326 & 0.8098 & 1.4085 & 1.2523 \\
\hline
\end{tabular}

In comparison with [6], the OT of relays is decreased by using the proposed Fuzzy logic in this paper. The convergence diagrams of the coefficients of the proposed OF are shown in Fig. 6. Using the Fuzzy logic and defining the appropriate rules play an important role in proper determination of $\mathrm{OF}$ parameters instead of the trial and error approach. This leads to better performance and more acceptable answers of OF. Defining the appropriate rules for the coefficients fuzzification leads to proper convergence and acceptable answers for the coefficients by which the OT of the relays and the number of miscoordination have been minimized. The results confirm that the proposed method not only has no limitation for using the diverse characteristic curves but also determine their coefficients such that the best answer is achieved.

\section{Scenario 3}

In order to validate the better performance of the proposed method, it is compared with diverse optimization methods including DE, IPM-GA, IPM-DE, IPM-branch and bound method (IPM-BBM), and IPM-IPM [19]. In this regard, the 6bus test system is used, the data of which can be found in [19]. There are 22 OC relays with the characteristic curves of IEC normally inverse. 52 pairs of primary and backup relays are identified in this network. The values of maximum load current, minimum fault current, and maximum fault current 
flowing through each relay can be found in [19]. The current transformers ratios for each relay and the close-in fault current value for each pair of relays have been presented in [19]. The TSM values of the relays are considered as continuous variables changing from 0.1 to 1.1 . In addition, it is assumed that the PSM values of the relays are discrete variables varying from 0.5 to 2 in the step of 0.25 .

TABLE VII

THE Proposed OF COEFFICIENTS IN THE SECOND SCENARIO

\begin{tabular}{|c|c|}
\hline Weighting Factor & Value \\
\hline$a_{1}$ & 0.2204 \\
\hline$a_{2}$ & 0.1573 \\
\hline$a_{3}$ & 0.0709 \\
\hline$a_{4}$ & 0.5015 \\
\hline
\end{tabular}
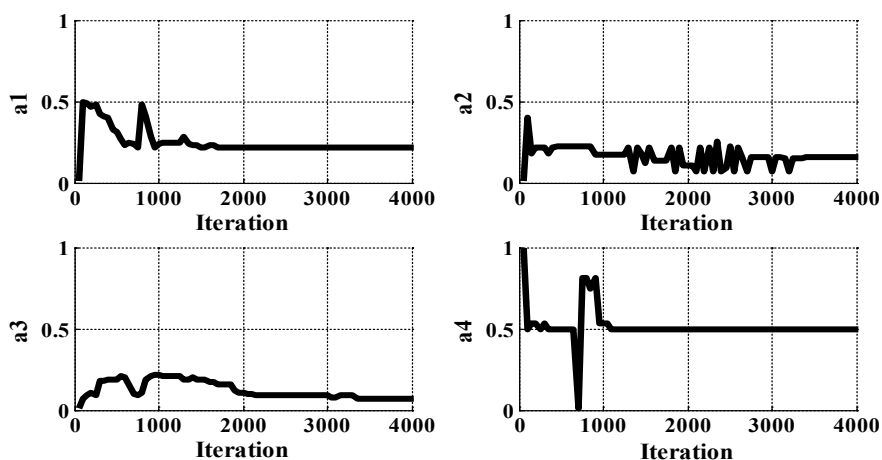

Fig. 6. Convergence of OF's coefficients in the second scenario

The results of comparison of the proposed method with other ones in [19] are listed in Table XI. These results include the optimal TSM and PSM values as well as the total operation time of the relays. The method in [19] chooses the best answer among the ones resulted from 100 simulations. Furthermore, the weighting factors of objective function are determined by a trial and error approach. On the other hand, the simulation results of the proposed algorithm in this paper are obtained just by one simulation and the algorithm by itself calculates the weighting factors of the objective function effectively. The values of these coefficients are listed in Table $X$. These values are calculated based on the defined fuzzy rules in this paper. Based on the presented results in the last row of Table XI, the operation time of OC relays (i.e., $\sum_{i=1}^{22} t_{o p, i}$ ) for the proposed method is less than the other ones while there is no miscoordination among the pairs of primary and backup relays.

\section{CONCLUSION}

In this paper, a Fuzzy-GA based method with a new OF has been proposed for optimal coordination of OC relays in interconnected networks. This method solves both miscoordination problems as well as the large OT of relays. Moreover, both discrete and continuous TSMs can be directly obtained through the proposed OF. PSM and the coefficients of characteristic curves of OC relays are other variables of the presented OF. These coefficients can be set in modern digital relays and this fact can result in better coordination and less OT. It confirms that the proposed method is capable to use any kind of characteristic curves. In addition, the weighting factors of OF are determined by the Fuzzy logic process in order to overcome the drawbacks existing in previous works for finding these factors. The proposed method is tested on three different networks including the 6-bus, 8-bus, and IEEE 30-bus test systems. The results of the proposed method have been compared with the different previous ones. The simulation results approve the effectiveness and accuracy of the proposed method because of its new expression for OF and its comprehensive variables.

TABLE IX

$\triangle T$ FOR EACH PAIR OF RELAYS IN THE SECOND SCENARIO

\begin{tabular}{|c|c|c|c|c|c|c|c|}
\hline 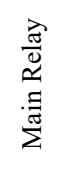 & 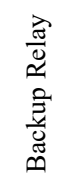 & 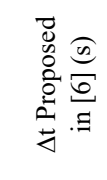 & 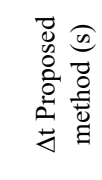 & 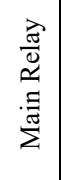 & 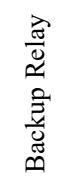 & 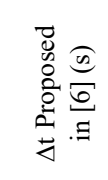 & 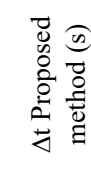 \\
\hline 1 & 21 & 0.2829 & 0.1161 & 10 & 29 & 0.4748 & 0.0121 \\
\hline 1 & 22 & -0.0223 & 0.0281 & 9 & 31 & 3.2547 & 0.0017 \\
\hline 2 & 22 & -0.0775 & 0.0395 & 11 & 29 & 0.548 & 0.1034 \\
\hline 3 & 21 & 0.0573 & 0.0029 & 10 & 31 & 3.2886 & 0.0404 \\
\hline 23 & 2 & 0.3652 & 0.1876 & 11 & 30 & 0.2082 & 0.2208 \\
\hline 5 & 2 & 0.2524 & 0.0854 & 12 & 10 & 0.1018 & 0.1073 \\
\hline 21 & 25 & 0.2 & 0.0507 & 30 & 32 & -0.0997 & 0.0126 \\
\hline 13 & 2 & 0.1378 & 0.1113 & 32 & 11 & 0.0188 & 0.3939 \\
\hline 21 & 33 & 0.0165 & 0.0819 & 31 & 12 & 0.0251 & 0.1386 \\
\hline 4 & 3 & -0.0145 & 0.3203 & 14 & 11 & 0.059 & 0.4679 \\
\hline 22 & 24 & -0.1701 & 0.0117 & 31 & 34 & -0.0144 & 0.2493 \\
\hline 23 & 25 & 0.2587 & 0.0872 & 14 & 12 & 0.000 & 0.0231 \\
\hline 23 & 33 & 0.0752 & 0.1186 & 32 & 34 & -0.0848 & 0.0606 \\
\hline 29 & 4 & 0.8823 & 0.0301 & 15 & 13 & 0.4422 & 0.0033 \\
\hline 24 & 9 & -0.039 & 0.099 & 33 & 35 & -0.085 & 0.001 \\
\hline 6 & 5 & 0.0015 & 0.0089 & 35 & 14 & -0.0224 & 0.0885 \\
\hline 25 & 26 & 0.016 & -0.0113 & 34 & 15 & 0.2844 & 0.0052 \\
\hline 5 & 33 & -0.037 & 0.0167 & 16 & 14 & 0.000 & 0.0653 \\
\hline 13 & 25 & 0.0319 & 0.0112 & 34 & 36 & -0.2149 & 0.0143 \\
\hline 7 & 6 & 0.000 & 0.0259 & 16 & 15 & 0.3037 & 0.0842 \\
\hline 26 & 27 & 0.2051 & -0.3191 & 35 & 36 & -0.2126 & 0.1166 \\
\hline 28 & 7 & -0.0211 & 0.0177 & 17 & 16 & 0.0305 & 0.1985 \\
\hline 27 & 8 & 0.1546 & 0.0046 & 18 & 16 & -0.082 & 0.0475 \\
\hline 8 & 29 & 0.3273 & 0.1057 & 36 & 37 & 0.9958 & 0.0446 \\
\hline 9 & 28 & 0.008 & 0.032 & 17 & 37 & 1.0094 & 0.3121 \\
\hline 8 & 30 & -0.0186 & 0.2178 & 19 & 18 & 0.0067 & 0.0323 \\
\hline 10 & 28 & 0.000 & 0.0675 & 20 & 18 & -0.0223 & 0.0053 \\
\hline 8 & 31 & 3.1065 & 0.1309 & 38 & 19 & 0.7395 & 0.1094 \\
\hline 11 & 28 & 0.0717 & 0.159 & 39 & 20 & 0.0525 & 0.547 \\
\hline 9 & 30 & 0.1385 & 0.0884 & & & & \\
\hline
\end{tabular}

TABLE X

THE PROPOSED OF'S COEFFICIENTS IN THE THIRD SCENARIO

\begin{tabular}{|c|c|}
\hline Coefficient & Value \\
\hline$a_{1}$ & 0.52 \\
\hline$a_{2}$ & 0.07 \\
\hline$a_{3}$ & 0.07 \\
\hline$a_{4}$ & 0.3 \\
\hline
\end{tabular}

\section{REFERENCES}

[1] A. S. Noghabi, J. Sadeh, and H. R. Mashhadi, "Considering different network topologies in optimal overcurrent relay coordination using a hybrid GA," IEEE Trans. Power Delivery, vol. 24, pp.1857-1863, Oct. 2009.

[2] P. P. Bedekar, and S. R. Bhide, "Optimum coordination of directional overcurrent relays using the hybrid GA-NLP approach," IEEE Trans. Power Delivery, vol. 26, pp. 109-119, Jan. 2011. 
[3] C. W. So, and K. K. Li, "Overcurrent relay coordination by evolutionary programming," Elect. Power Syst. Res., vol. 53, pp. 83-90, Feb. 2000.

[4] R. Mohammadi, H. A. Abyaneh, H. M. Rudsari, S. H. Fathi, and H. Rastegar, "Overcurrent relays coordination considering the priority of constraints," IEEE Trans. Power Delivery, vol. 26, pp. 1927-1938, Jul. 2011.

[5] A. H. Abyaneh, M. Al-Dabbagh, H. K. Karegar, S. H. H. Sadeghi, and R. A. J. Khan, "A new optimal approach for coordination of overcurrent relays in interconnected power systems," IEEE Trans. Power Delivery, vol. 18, pp. 430-435, Apr. 2003

[6] F. Razavi, H. A. Abyaneh, M. Al-Dabbagh, R. Mohammadi, and H. Torkaman, "A new comprehensive genetic algorithm method for optimal overcurrent relays coordination," Elect. Power Syst. Res., vol. 78, pp. 713-720, Apr. 2008.

[7] H. K. Kargar, H. A. Abyaneh, V. Ohis, and M. Meshkin, "Preprocessing of the optimal coordination of overcurrent relays," Elect. Power Syst. Res., vol. 75, pp. 134-141, Aug. 2005.

[8] T. Amraee, "Coordination of Directional Overcurrent Relays Using Seeker Algorithm" IEEE Trans. Power Delivery, vol. 27, pp. 14151422, Apr. 2012

[9] C. A. C. Salazara, A. C. Enríqueza, and S. E. Schaeffer, "Directional overcurrent relay coordination considering non-standardized time curves" Elect. Power Syst. Res., vol. 122, pp. 42-49, May 2015.

[10] C. W. So, and K. K. Li, "Intelligent method for protection coordination," in Proc. 2004 IEEE International Conf. of Electric Utility Deregulation Restructuring and Power Technology, pp. 378-382.

[11] R. Mohammadi, H. A. Abyaneh, F. Razavi, M. Al-Dabbagh, S. H. H. Sadeghi, "Optimal relays coordination efficient method in interconnected power systems" Journal of ELECTRICAL ENGINEERING, vol. 61, pp. 75-83, Mar. 2010.

[12] P. P. Bedekar, and S. R. Bhide "Optimum coordination of overcurrent relay timing using continuous genetic algorithm," Expert Systems with Appl., vol. 38, pp. 11286-11292, Sep. 2011.

[13] C. W. So, and K. K. Li, "Time coordination method for power system protection by evolutionary algorithm," IEEE Trans. Ind. Appl. vol. 36, pp. 1235-1240, Sep. 2000.

[14] F. Adelnia, Z. Moravej, and M. Farzinfar, "A new formulation for coordination of directional overcurrent relays in interconnected networks," Int. Trans. Electr. Energy System, vol. 25, pp. 120-137, Jan. 2015.
[15] T. Keil, and J. Jäger, "Advanced Coordination Method for Overcurrent Protection Relays Using Nonstandard Tripping Characteristics" IEEE Trans. Power Delivery, vol. 23, pp. 52-57, Jun. 2008.

[16] M. N. Alam, B. Das, and V. Pant, "A comparative study of metaheuristic optimization approaches for directional overcurrent relays coordination" Elect. Power Syst. Res., vol. 128, pp. 39-52, Nov. 2015.

[17] K. A. Saleh, H. H. Zeineldin, A. Al-Hinai, and E. F. El-Saadany, "Optimal coordination of directional overcurrent relays using a new time-current-voltage characteristic" IEEE Trans. Power Delivery, vol. 30, pp. 537-544, Aug. 2014.

[18] Z. Moraveja, F. Adelniaa, and F. Abbasi, "Optimal coordination of directional overcurrent relays using NSGA-II" Elect. Power Syst. Res., vol. 119, pp. 228-236, Feb. 2015.

[19] M. N. Alam, B. Das, and V. Pant, "An interior point method based protection coordination scheme for directional overcurrent relays in meshed networks" Int. Journal of Electr. Power \& Energy Systems, vol. 81, pp. 153-164, Oct. 2016.

[20] J. Jäger, R. Lubiatowski, G. Ziegler and R. Krebs, "Protection security assessment for large power systems," PowerTech, 2009 IEEE Bucharest, Bucharest, 2009, pp. 1-6.

[21] J. Jäger, T. Keil, A. Dienstbier, P. Lund and R. Krebs, "Network security assessment - An important task in distribution systems with dispersed generation," Electricity Distribution - Part 1, CIRED, 2009, pp. 1-4.

[22] M. Ezzeddine, R. Kaczmarek, "A novel method for optimal coordination of directional overcurrent relays considering their available discrete settings and several operation characteristics," Elect. Power Syst. Res., vol. 81, pp. 1475-1481, Jul. 2011.

[23] Available: http://new.abb.com/substationautomation/products/protection-control/relion-product-family/relion615-series

[24] D. Solati Alkaran, M. R. Vatani, M. J. Sanjari, and G. B. Gharehpetian, "Overcurrent relays coordination in interconnected networks using accurate analytical method and based on determination of fault critical point," IEEE Trans. Power Delivery, vol. 30, pp. 870-877, Apr. 2015.

[25] "Power system test scenario archive," available: $\mathrm{http}: / /$ www.ee.washington.edu/research/pstca/

[26] IEC Standard for Single Input Energizing Quantity Measuring Relays with Dependent Specified Time, IEC Standard 255-4, 1976.

TABLE XI

TSM AND PSM FOR THE RELAYS IN THE THIRD SCENARIO

\begin{tabular}{|c|c|c|c|c|c|c|c|c|c|c|c|c|}
\hline \multirow{2}{*}{ Relay No. } & \multicolumn{2}{|c|}{ DE } & \multicolumn{2}{|c|}{ IPM-GA } & \multicolumn{2}{c|}{ IPM-DE } & \multicolumn{2}{c|}{ IPM-BBM } & \multicolumn{2}{c|}{ IPM-IPM } & \multicolumn{2}{c|}{ Proposed Method } \\
\cline { 2 - 15 } & TSM & PSM & TSM & PSM & TSM & PSM & TSM & PSM & TSM & PSM & TSM & PSM \\
\hline 1 & 0.1061 & 2 & 0.1019 & 2 & 0.102 & 2 & 0.1019 & 2 & 0.1019 & 2 & 0.102 & 2 \\
\hline 2 & 0.1001 & 1.75 & 0.1102 & 1.25 & 0.1001 & 1.5 & 0.1 & 1.5 & 0.1081 & 1.25 & 0.1079 & 1.25 \\
\hline 3 & 0.1499 & 1.5 & 0.1443 & 1.5 & 0.1331 & 1.75 & 0.1313 & 1.75 & 0.1314 & 1.75 & 0.131 & 1.75 \\
\hline 4 & 0.1 & 0.5 & 0.1 & 0.5 & 0.1001 & 0.5 & 0.1 & 0.5 & 0.1 & 0.5 & 0.1 & 0.5 \\
\hline 5 & 0.1 & 2 & 0.1092 & 1.75 & 0.1001 & 2 & 0.1 & 2 & 0.107 & 1.75 & 0.1069 & 1.75 \\
\hline 6 & 0.1008 & 2 & 0.1098 & 1.75 & 0.1 & 2 & 0.1 & 2 & 0.1 & 2 & 0.1 & 2 \\
\hline 7 & 0.1124 & 2 & 0.1208 & 1.75 & 0.1123 & 2 & 0.1074 & 2 & 0.1076 & 2 & 0.1075 & 2 \\
\hline 8 & 0.218 & 0.75 & 0.1921 & 1 & 0.1736 & 1.25 & 0.1734 & 1.25 & 0.1741 & 1.25 & 0.1686 & 1.25 \\
\hline 9 & 0.1001 & 1.75 & 0.1 & 1.5 & 0.1001 & 1.5 & 0.1 & 1.5 & 0.1 & 1.5 & 0.1 & 1.5 \\
\hline 10 & 0.1001 & 1.75 & 0.1082 & 1.25 & 0.1 & 1.5 & 0.1054 & 1.25 & 0.1055 & 1.25 & 0.1046 & 1.25 \\
\hline 11 & 0.102 & 1.25 & 0.1037 & 1.25 & 0.1 & 1.5 & 0.1015 & 1.25 & 0.1015 & 1.25 & 0.1014 & 1.25 \\
\hline 12 & 0.1001 & 1.5 & 0.1022 & 1.25 & 0.1 & 1.5 & 0.1 & 1.5 & 0.1022 & 1.25 & 0.1022 & 1.25 \\
\hline 13 & 0.1109 & 2 & 0.1095 & 2 & 0.1088 & 2 & 0.1087 & 2 & 0.1089 & 2 & 0.1072 & 2 \\
\hline 14 & 0.1242 & 2 & 0.1213 & 2 & 0.1196 & 2 & 0.1195 & 2 & 0.1199 & 2 & 0.1198 & 2 \\
\hline 15 & 0.1227 & 2 & 0.1604 & 1.25 & 0.1435 & 1.5 & 0.1434 & 1.5 & 0.157 & 1.25 & 0.1571 & 1.25 \\
\hline 16 & 0.1508 & 2 & 0.1579 & 1.75 & 0.1507 & 2 & 0.1507 & 2 & 0.1444 & 2 & 0.1443 & 2 \\
\hline 17 & 0.1001 & 1.5 & 0.1068 & 1.25 & 0.1 & 1.5 & 0.1 & 1.25 & 0.106 & 1.25 & 0.1059 & 1.25 \\
\hline 18 & 0.1314 & 2 & 0.129 & 2 & 0.1277 & 2 & 0.1277 & 2 & 0.1279 & 2 & 0.1251 & 2 \\
\hline 19 & 0.1001 & 2 & 0.1 & 2 & 0.1 & 2 & 0.1 & 2 & 0.1024 & 1.75 & 0.1024 & 1.75 \\
\hline 20 & 0.1229 & 2 & 0.1279 & 1.75 & 0.1181 & 2 & 0.1181 & 2 & 0.1167 & 2 & 0.116 & 2 \\
\hline 21 & 0.1168 & 2 & 0.1275 & 1.75 & 0.1146 & 2 & 0.1146 & 2 & 0.1147 & 2 & 0.1129 & 2 \\
\hline 22 & 0.1378 & 1.5 & 0.1401 & 1.5 & 0.1257 & 1.75 & 0.1257 & 1.75 & 0.1371 & 1.5 & 0.1371 & 1.5 \\
\hline$\sum_{i=1}^{22} t_{\text {op }, i}$ & 7.8086 & & 7.7024 & & 7.6499 & & 7.5487 & & 7.5482 & & 7.5059 \\
\hline
\end{tabular}


TABLE VIII

PSM, TSM, OT, AND THE COEFFICIENTS OF CHARACTERISTIC CURVES FOR THE RELAYS IN THE SECOND SCENARIO

\begin{tabular}{|c|c|c|c|c|c|c|c|c|c|c|c|c|}
\hline & \multicolumn{6}{|c|}{ Proposed Method in [6] } & \multicolumn{6}{|c|}{ Proposed Method } \\
\hline Relay & PSM (A) & TSM (s) & OT (s) & A & B & $\mathrm{C}$ & PSM (A) & TSM (s) & OT (s) & A & B & $\mathrm{C}$ \\
\hline 1 & 132.5025 & 1.36 & 0.0006 & 146.663 & 2.686 & 0.870 & 176.67 & 1.71 & 0.0861 & 93.451 & 1.681 & 0.686 \\
\hline 2 & 617.565 & 1.26 & 0.0555 & 112.9513 & 2.494 & 0.274 & 308.7825 & 0.55 & 0.0742 & 126.397 & 1.782 & 0.520 \\
\hline 3 & 128.7975 & 1.6 & 0.223 & 110.380 & 1.421 & 0.786 & 85.865 & 0.7 & 0.1965 & 140.753 & 1.218 & 0.683 \\
\hline 4 & 86.19 & 0.57 & 0.8413 & 146.494 & 1.143 & 0.594 & 129.285 & 0.68 & 0.2081 & 87.38 & 1.561 & 0.576 \\
\hline 5 & 136.37 & 0.58 & 0.1132 & 134.480 & 1.574 & 0.424 & 204.555 & 1.6 & 0.105 & 65.981 & 1.846 & 0.880 \\
\hline 6 & 78.65 & 1.13 & 0.23 & 60.067 & 1.477 & 0.572 & 78.65 & 0.75 & 0.2956 & 112.808 & 1.469 & 0.549 \\
\hline 7 & 242.19 & 0.19 & 0.17 & 161.448 & 2.158 & 0.654 & 80.73 & 0.81 & 0.2473 & 141.692 & 1.751 & 0.721 \\
\hline 8 & 106.145 & 0.72 & 0.2361 & 154.027 & 1.300 & 0.263 & 212.29 & 0.15 & 0.0754 & 80.971 & 1.259 & 0.352 \\
\hline 9 & 188.5 & 0.88 & 0.078 & 117.491 & 1.762 & 0.438 & 150.8 & 1.23 & 0.2039 & 150.386 & 1.583 & 0.807 \\
\hline 10 & 406.64 & 1.64 & 0.0873 & 156.923 & 2.342 & 0.257 & 406.64 & 0.97 & 0.1683 & 74.022 & 1.776 & 0.632 \\
\hline 11 & 290.355 & 1.91 & 0.0148 & 30.168 & 2.192 & 0.804 & 145.1775 & 1.51 & 0.0774 & 94.721 & 1.684 & 0.725 \\
\hline 12 & 76.635 & 1.4 & 0.1702 & 177.417 & 1.671 & 0.555 & 25.545 & 0.24 & 0.3001 & 76.325 & 0.755 & 0.480 \\
\hline 13 & 159.25 & 1.31 & 0.2277 & 127.341 & 1.651 & 0.502 & 191.1 & 1.77 & 0.079 & 152.593 & 2.133 & 0.450 \\
\hline 14 & 214.695 & 1.61 & 0.0479 & 73.513 & 2.158 & 0.329 & 107.3475 & 0.65 & 0.1295 & 142.629 & 1.524 & 0.872 \\
\hline 15 & 72.735 & 1.59 & 0.2092 & 55.905 & 1.520 & 0.409 & 36.3675 & 0.1 & 0.2304 & 185.738 & 0.940 & 0.484 \\
\hline 16 & 78.39 & 1.99 & 0.0409 & 142.473 & 2.051 & 0.183 & 52.26 & 0.83 & 0.1338 & 79.300 & 1.314 & 0.480 \\
\hline 17 & 47.645 & 0.99 & 0.0002 & 33.902 & 2.600 & 0.782 & 95.29 & 0.54 & 0.0077 & 158.459 & 2.371 & 0.481 \\
\hline 18 & 106.73 & 0.76 & 0.1123 & 105.893 & 2.080 & 0.654 & 53.365 & 0.44 & 0.1583 & 129.673 & 1.528 & 0.721 \\
\hline 19 & 178.1 & 0.69 & 0.0119 & 133.683 & 2.656 & 0.309 & 71.24 & 0.57 & 0.0262 & 55.257 & 1.655 & 0.901 \\
\hline 20 & 202.3125 & 0.09 & 0.0412 & 49.079 & 1.443 & 0.366 & 242.775 & 0.38 & 0.0534 & 76.137 & 2.057 & 0.635 \\
\hline 21 & 411.71 & 0.11 & 0.059 & 65.484 & 1.997 & 0.756 & 205.855 & 0.52 & 0.0392 & 81.257 & 2.252 & 0.724 \\
\hline 22 & 257.595 & 0.1 & 0.0581 & 41.207 & 1.621 & 0.922 & 128.7975 & 0.3 & 0.1025 & 84.705 & 1.656 & 0.224 \\
\hline 23 & 18.655 & 1.46 & 0.001 & 80.999 & 1.904 & 0.553 & 18.655 & 0.89 & 0.003 & 96.904 & 1.666 & 0.699 \\
\hline 24 & 129.285 & 1.97 & 0.021 & 67.859 & 2.202 & 0.879 & 86.19 & 0.33 & 0.1375 & 107.311 & 1.267 & 0.372 \\
\hline 25 & 170.4625 & 0.42 & 0.1831 & 47.660 & 1.707 & 0.338 & 68.185 & 0.5 & 0.1155 & 125.421 & 1.717 & 0.320 \\
\hline 26 & 78.65 & 0.8 & 0.272 & 35.323 & 1.210 & 0.442 & 31.46 & 0.27 & 0.2209 & 99.245 & 1.009 & 0.494 \\
\hline 27 & 121.095 & 0.64 & 0.5036 & 163.633 & 1.479 & 0.365 & 242.19 & 1.53 & 0.0594 & 100.654 & 2.696 & 0.315 \\
\hline 28 & 159.2175 & 0.85 & 0.0577 & 55.035 & 2.503 & 0.611 & 159.2175 & 0.34 & 0.1055 & 116.185 & 2.215 & 0.601 \\
\hline 29 & 113.1 & 0.99 & 0.4981 & 66.605 & 1.523 & 0.408 & 226.2 & 0.27 & 0.2603 & 28.307 & 1.348 & 0.325 \\
\hline 30 & 203.32 & 0.07 & 0.0231 & 126.149 & 1.970 & 0.381 & 609.96 & 0.02 & 0.0716 & 30.751 & 1.147 & 0.446 \\
\hline 31 & 145.1775 & 0.68 & 0.0175 & 133.539 & 2.217 & 0.354 & 96.785 & 0.14 & 0.0141 & 39.012 & 1.397 & 0.293 \\
\hline 32 & 51.09 & 0.64 & 0.0876 & 155.327 & 1.521 & 0.555 & 51.09 & 1.57 & 0.2025 & 137.808 & 1.508 & 0.498 \\
\hline 33 & 159.25 & 0.9 & 0.0722 & 171.593 & 2.769 & 0.553 & 191.1 & 0.14 & 0.1364 & 88.395 & 1.746 & 0.821 \\
\hline 34 & 107.3475 & 1.47 & 0.0595 & 124.748 & 2.307 & 0.789 & 71.565 & 1.35 & 0.2125 & 46.133 & 1.461 & 0.519 \\
\hline 35 & 60.6125 & 1.3 & 0.0572 & 63.561 & 1.631 & 0.823 & 36.3675 & 1.33 & 0.1104 & 173.371 & 1.537 & 0.597 \\
\hline 36 & 78.39 & 2 & 0.0123 & 71.251 & 2.745 & 0.433 & 26.13 & 0.18 & 0.2747 & 98.770 & 0.927 & 0.637 \\
\hline 37 & 133.4125 & 1.82 & 0.5934 & 139.721 & 1.860 & 0.580 & 53.365 & 0.41 & 0.318 & 164.784 & 1.284 & 0.260 \\
\hline 38 & 80.925 & 1.18 & 0.0589 & 62.856 & 2.862 & 0.235 & 80.925 & 0.38 & 0.1068 & 21.859 & 1.749 & 0.728 \\
\hline 39 & 42.51 & 0.26 & 0.0215 & 94.203 & 2.009 & 0.722 & 42.51 & 0.43 & 0.0398 & 117.436 & 2.040 & 0.485 \\
\hline Sum & & 39.93 & 5.5681 & & & & & 27.08 & 5.3868 & & & \\
\hline
\end{tabular}

